\title{
Nuclear Shapes in Complex Fission Phenomena
}

\author{
D. N. Poenaru, ${ }^{*, a, b, c}$ W. Greiner, ${ }^{b}$ Y. Nagame, ${ }^{\mathbf{c}}$ and R. A. Gherghescu ${ }^{\mathrm{a}, \mathrm{b}}$ \\ ${ }^{a}$ National Institute of Physics \& Nuclear Engineering, PO Box MG-6, RO-76900 Bucharest-Magurele, \\ Romania \\ ${ }^{\mathrm{b}}$ Institut für Theoretische Physik der J. W. Goethe Universität, Pf 111932, D-60054 Frankfurt am Main, \\ Germany \\ 'Japan Atomic Energy Research Institute, Tokai, Ibaraki 319-1195, Japan
}

Received: November 13, 2001; In Final Form: November 13, 2001

\begin{abstract}
We present a method for finding very general reflection asymmetric equilibrium (saddle-point) nuclear shapes, as solutions of an integro-differential equation, without giving a shape parametrization. By introducing phenomenological shell corrections one obtains minima of deformation energy for binary fission of parent nuclei ${ }^{238} \mathrm{U},{ }^{232,228} \mathrm{Th}$ at a non-zero mass asymmetry, leading to the same mass number of the heavy fragment $A_{1}=125$. Applications for fission into more than two and three fragments are also illustrated.
\end{abstract}

\section{Introduction}

The permanent distortion from a sphere of a given nucleus in its ground state or the change of deformation during a nuclear process (e.g. fission or fusion) may be described in terms of collective coordinates. One has to choose a function with a number of parameters as low as possible, which in the same time allows to determine the most important shapes of the nuclear surface. There are many such parametrizations described in the literature (see various chapters of the books ${ }^{1,2}$ and references therein). The surface equation, determined by a set of deformation coordinates, is frequently used to calculate the potential energy surfaces (PES) which in turn can be applied to find the nuclear deformations and fission barriers, to explain shape isomers, to obtain indications about mass asymmetry in fission, to calculate half-lives against various decay modes or to study multidimensional tunneling, ${ }^{3}$ to extend the nuclear chart in the region of superheavy nuclei, etc.

Since the early days of development of nuclear fission, the shapes during the deformation process from one parent nucleus to the final fragments, have been intensively studied either statically or dynamically. ${ }^{6,7}$ Within a liquid drop model (LDM) all nuclear shapes in the ground-state are spherical and the fission fragment mass distributions are symmetric, in contrast to reality. Permanent nuclear deformations and fission fragment mass asymmetry can be explained by combining the collective (liquid drop-like) and single particle properties in the framework of macroscopic-microscopic Strutinsky's method. By using the two center shell model ${ }^{8}$ to describe the single-particle states, one can follow the shell structure all the way from the original nucleus, over the potential barriers, up to the final stage of separated fragments. Particularly important points on a potential energy surface are those corresponding to the ground-state, ${ }^{9}$ saddle-point(s) ${ }^{10,11}$ and scission point. ${ }^{12,13}$

The progress in understanding the connection between scission configurations and the binary fission mechanisms and mass yield was recently reviewed. ${ }^{14}$ A systematic analysis of a large body of experimental data (see also Reference 15) on total kinetic energy (TKE) of fission fragments confirmed the existence of two fission modes in the region of actinides: one with low TKE components and elongated scission shapes leading to a symmetric mass yield, and the other one with high TKE components and compact shapes connected to a shell-influenced asymmetric yield. Two kinds of fission paths were assumed on this basis: with lower barrier going through reflection asymmetric compact configurations, and with higher barrier and sym-

*Corresponding author. E-mail: poenaru@ifin.nipne.ro. FAX: +401-457-4440. metric scission configurations. The invariance of scission deformation $\beta$ was demonstrated ${ }^{16}$ and new formulas replacing Viola systematics were given. The deformation was defined by $\beta=D\left(A_{1}, A_{2}\right) / D_{0}\left(A_{1}, A_{2}\right)$ where $D=Z_{1} Z_{2} e^{2} / T K E\left(A_{1}, A_{2}\right)$ is the distance between charge centers of the fragments and $D_{0}$ is the distance between centers of two touching spherical fragments with radii $R_{i}=1.17 A_{i}^{1 / 3} \mathrm{fm}$. For the first time it was experimentally found ${ }^{17}$ that scissioning nuclei with a given elongation lead to a given shape of the fragment mass yield curve. By including into analysis the region of heaviest nuclei with measured TKE (heavy Fm, Bk, No, Cf, Lr, and Rf isotopes) the existence of three types of shape elongations was clearly shown: LDM governed mass symmetric elongated shape $(\beta \simeq 1.65)$; mass asymmetric deformation $(\beta \simeq 1.53)$, and shell-influenced mass symmetric deformation $(\beta \simeq 1.43)$.

Important achievements have been also reached in another experimental field including rare phenomena, where new characteristics of fission process ${ }^{18}$ and new decay modes ${ }^{19}(\alpha$ and ${ }^{10} \mathrm{Be}$ accompanied cold fission of ${ }^{252} \mathrm{Cf}$, double fine structure and triple fine structure in binary and ternary fission, respectively) were discovered during the last years by using triple- $\gamma$ coincidences in a large array of Germanium Compton-suppressed detectors (the famous GAMMASPHERE). The increased sensitivity of such measurements could allow to detect in the future other rare phenomena, as multicluster accompanied fission ${ }^{20}$ and to continue to search for new kinds of quasimolecular states. ${ }^{21}$

In a statical approach, the equilibrium nuclear shapes are usually obtained by minimizing the energy functional on a certain class of trial functions representing the surface equation. The simplest shape one can imagine, still allowing to describe essential features of a cluster decay process is the spherical one. ${ }^{2}$ Even for a particle-accompanied fission one can imagine ${ }^{21}$ a single-deformation parametrization based on spherical shapes.

The purpose of this paper is to present a method allowing to obtain a very general reflection asymmetric saddle-point shape as a solution of an integro-differential equation without a shape parametrization apriori introduced. This equation was derived by minimizing the potential energy with constraints (constant volume and given deformation parameter). The method allows to obtain straightforwardly the axially-symmetric surface shape for which the liquid drop energy, $E_{\mathrm{LDM}}=E_{s}+E_{C}$, is minimum. By adding the shell corrections $\delta E$ to the LDM deformation energy, $E_{\text {def }}=E_{\mathrm{LDM}}+\delta E$, we succeeded to obtain minima at a finite value of the mass-asymmetry parameter. We use a phenomenological shell correction. Results for binary fission of parent nuclei ${ }^{238} \mathrm{U}$ and ${ }^{232,228} \mathrm{Th}$ are presented. The minima of the saddle-point energy occur at nonzero mass asymmetry parameters which happens to lead to the same mass number of the 
heavy fragment, $A_{1}=125$, for all fissioning nuclei mentioned above. Applications for fission into more than two and three fragments are also illustrated.

\section{Saddle Point}

Let us consider a nuclear system with a shape specified by a set of $n$ generalized coordinates $\left\{q_{i}\right\}$. For an equilibrium (ground-state or saddle-point) shape ${ }^{22}$ the deformation energy $E=E\left(q_{1}, q_{2}, \ldots, q_{n}\right)$ has an extremum, defined by $\partial E / \partial q_{i}=0$, $(i=1,2, \ldots, n)$. In a LDM the ground state, characterized by the lowest minimum of the potential energy, always corresponds to a spherical shape. One may define a fission valley on the PES in a multidimensional space of deformation parameters, as a conditional minimum $\partial E / \partial q_{k}=0,(\mathrm{i}=1,2, \ldots, \mathrm{k}-1, \mathrm{k}+1, \ldots, \mathrm{n})$ with the constraint $q_{k}=q_{k}^{0}$ for different values $q_{k}^{0}$. The maximum value on this minimum energy determines the saddle-point position, at which all eigenvalues of the symmetric curvature matrix $K_{i j}=\partial^{2} E(q) /\left(\partial q_{i} \partial q_{j}\right)$ have a positive sign, except one. For two deformation coordinates ${ }^{23}$ at the saddle point

$$
\left|\begin{array}{cc}
\frac{\partial^{2} E}{\partial q_{1}^{2}} & \frac{\partial^{2} E}{\partial q_{1} \partial q_{2}} \\
\frac{\partial^{2} E}{\partial q_{2} \partial q_{2}} & \frac{\partial^{2} E}{\partial q_{2}^{2}}
\end{array}\right|<0 .
$$

The simplest LDM potential energy vs. one deformation parameter along the fission path is a smooth curve with a minimum at the ground state and a maximum at the saddle point. Then it decreases continuously going through the scission point, down to the self energy of the fragments at infinite separation distance. By representing this quantity for a heavy nucleus vs. the mass asymmetry coordinate in a transverse direction at the saddle point we also get a smooth curve with a minimum between two maxima, called Businaro-Gallone mountains. The saddle point is lying on the bottom of the valley separating these mountains.

A conditional saddle point is defined by imposing one or several constraints. Of particular interest in fission is the constraint of determined mass asymmetry, $\eta=\eta_{0}$. If $\eta$ is one of the generalized coordinates the problem is simplified because one takes $\eta=$ constant. In general $\eta$ depends on $q$, and the generalization of the variational equation to the equilibrium with constraints leads to

$$
\frac{\partial E}{\partial q_{i}}+\lambda \frac{\partial \eta}{\partial q_{i}}=0, \quad i=1,2, \ldots, n
$$

where $\lambda$ is a Lagrange multiplier.

There is no need to consider any reflection asymmetry or nonaxiality in the calculation of saddle point shapes within LDM, because the energy increases in the presence of both kinds of deviations from symmetry. The parametrization of Legendre polynomial expansion with even-order deformation parameters $\alpha_{2 n}$ up to $n=18$ was used. ${ }^{4}$ For low fissility, $X$, the saddle point shapes are very similar to two tangent spheres, hence it is more difficult to be described with a small number of deformation coordinates. By increasing fissility a neck develops between the two symmetrical fragments. The length increases up to $X=0.67$ and in the same time the neck radius becomes larger. The best accuracy was obtained at larger fissilities, close to $X=1$, for which the saddle point shapes are not very different from a single sphere.

\section{The Model}

We are looking for a nuclear surface equation with axial symmetry around the $z$ axis, expressed as $\rho=\rho(z)$ in cylindrical coordinates, which minimizes the potential energy of deformation with two constraints: volume conservation, and given deformation parameter, $\alpha$, assumed to be an adiabatic variable. With re- spect to spherical shape (superscript ${ }^{0}$ ), the deformation energy is defined as

$$
\begin{aligned}
E_{\mathrm{def}}(\alpha)-E^{0} & =\left(E_{s}-E_{s}^{0}\right)+\left(E_{C}-E_{C}^{0}\right)+\delta E-\delta E^{0} \\
& =E_{s}^{0}\left[B_{s}-1+2 X\left(B_{C}-1\right)\right]+\delta E-\delta E^{0}
\end{aligned}
$$

where $E_{s}^{0}=a_{s}\left(1-\kappa I^{2}\right) A^{2 / 3}$ and $E_{C}^{0}=a_{C} Z^{2} A^{-1 / 3}$ are energies corresponding to spherical shape and $I=(N-Z) / A$. The relative surface and Coulomb energies $B_{s}=E_{s} / E_{s}^{0}, B_{C}=E_{C} / E_{C}^{0}$ and the shell correction $\delta E(\alpha)$ are functions of the nuclear shape. The dependence on the neutron and proton numbers is contained in $E_{s}^{0}$, the fissility parameter $X=E_{C}^{0} /\left(2 E_{s}^{0}\right)=$ $\left[3 Z^{2} e^{2} /\left(5 R_{0}\right)\right] / 2\left[a_{s}\left(1-\kappa I^{2}\right) A^{2 / 3}\right]$, as well as in shell correction of the spherical nucleus $\delta E^{0}$. From a fit to experimental data on nuclear masses, quadrupole moments, and fission barriers, the following values of the parameters have been obtained: ${ }^{24} a_{s}=$ $17.9439 \mathrm{MeV}, \kappa=1.7826, a_{C}=3 e^{2} /\left(5 r_{0}\right)=0.7053 \mathrm{MeV}$. The radius of spherical nucleus is $R_{0}=r_{0} A^{1 / 3}$ with $r_{0}=1.2249 \mathrm{fm}$, and $e^{2}=1.44 \mathrm{MeV} \cdot \mathrm{fm}$ is the square of electron charge. Calculation of $\delta E$ will be outlined below.

The corresponding variational problem can be reduced to the following equation

$$
\rho \rho^{\prime \prime}-\rho^{\prime 2}-\left[\lambda_{1}+\lambda_{2}|z|+10 X V_{s}(z, \rho)\right] \rho\left(1+\rho^{\prime 2}\right)^{3 / 2}-1=0
$$

where $\rho^{\prime}=d \rho / d z, \rho^{\prime \prime}=d^{2} \rho / d z^{2}$, and $V_{s}$ is the Coulomb potential on the nuclear surface. We used the following relationships for the principal radii of curvature $\mathcal{R}_{1}=\tau \rho, \mathcal{R}_{2}{ }^{-1}=-\rho^{\prime \prime} / \tau^{3}$, in which $\tau^{2}=1+\rho^{\prime 2}$. In eq $4 \lambda_{1}$ and $\lambda_{2}$ are Lagrange multipliers corresponding to the constraints of volume conservation (or given mass asymmetry if the volume is conserved in each "half" of the nucleus) and determined value of deformation parameter $\alpha$, defined as the distance between centers of mass of the fragments lying at the left hand side and right hand side of the plane $z=0$, respectively: $\alpha=\left|z_{L}^{c}\right|+\left|z_{R}^{c}\right|$. This definition allows to reach all intermediate stages of deformation from one parent nucleus to two fragments by a continuous variation of its value. The position of separation plane, $z=0$, is given by the condition: $(d \rho / d z)_{z=0}=0$. Lengths are given in units of $R_{0}$, Coulomb potential in units of $Z e / R_{0}$, and energy in units of the surface energy $E_{s}^{0}$. One can calculate for every value of $\alpha$ the deformation energy $E_{\text {def }}(\alpha)$. The particular value $\alpha_{s}$ for which $d E_{\mathrm{def}}\left(\alpha_{s}\right) / d \alpha=0$ corresponds to the extremum, i.e. the shape function describes the saddle point (or the ground state), and the unconditional extremum of the energy is the fission barrier. It can also be obtained by taking $\lambda_{2}=0$ in eq 4 . The other surfaces (for $\alpha \neq \alpha_{s}$ ) are extrema only with condition $\alpha=$ constant. In this way one can compute the deformation energy function of two variables: elongation and mass asymmetry.

The Coulomb potential on the surface depends on the function $\rho(z)$, hence eq 4 is an integro-differential one, as $V_{s}$ is expressed by an integral on the nuclear volume. One has indeed, for the general case of non-axial symmetry in cylindrical coordinates $(\rho, \varphi, z)$

$$
E_{C}=\frac{\rho_{e}}{5} \int_{z_{1}}^{z_{2}} d z \int_{0}^{2 \pi}\left(\rho^{2}-\frac{z}{2} \frac{\partial \rho^{2}}{\partial z}\right) V d \varphi
$$

where $\rho=\rho(z, \varphi)$ is the nuclear surface equation, $z_{1}$ and $z_{2}$ are the intersections of the surface with $O z$ axis, and $V$ is the electrostatic potential on the nuclear surface.

For axial-symmetry the shape-dependent, dimensionless surface term is proportional to the surface area:

$$
B_{s}=\frac{E_{s}}{E_{s}^{0}}=\frac{d^{2}}{2} \int_{-1}^{+1}\left[y^{2}+\frac{1}{4}\left(\frac{d y^{2}}{d x}\right)^{2}\right]^{1 / 2} d x
$$

where $y=y(x)$ is the surface equation in cylindrical coordinates with $-1,+1$ intercepts on the symmetry axis, and $d=$ 
$\left(z_{2}-z_{1}\right) / 2 R_{0}$ is the seminuclear length in units of $R_{0}$. Similarly, for the Coulomb energy ${ }^{25}$ one has

$$
B_{c}=\frac{5 d^{5}}{8 \pi} \int_{-1}^{+1} d x \int_{-1}^{+1} d x^{\prime} F\left(x, x^{\prime}\right)
$$

$$
\begin{aligned}
& F\left(x, x^{\prime}\right)=a_{\rho}^{-1}\left\{y y_{1}[(K-2 D) / 3]\right. \\
& \times\left[2\left(y^{2}+y_{1}^{2}\right)-\left(x-x^{\prime}\right)^{2}+\frac{3}{2}\left(x-x^{\prime}\right)\left(\frac{d y_{1}^{2}}{d x^{\prime}}-\frac{d y^{2}}{d x}\right)\right]+K \\
& \quad \times\left\{y^{2} y_{1}^{2} / 3+\left[y^{2}-\frac{x-x^{\prime}}{2} \frac{d y^{2}}{d x}\right]\left[y_{1}^{2}-\frac{x-x^{\prime}}{2} \frac{d y_{1}^{2}}{d x^{\prime}}\right]\right\}
\end{aligned}
$$

$K, K^{\prime}$ are the complete elliptic integrals of the 1st and 2 nd kind

$$
K(k)=\int_{0}^{\pi / 2}\left(1-k^{2} \sin ^{2} t\right)^{-1 / 2} d t ; \quad K^{\prime}(k)=\int_{0}^{\pi / 2}\left(1-k^{2} \sin ^{2} t\right)^{1 / 2} d t
$$

and $a_{\rho}^{2}=\left(y+y_{1}\right)^{2}+\left(x-x^{\prime}\right)^{2}, k^{2}=4 y y_{1} / a_{\rho}^{2}, D=\left(K-K^{\prime}\right) / k^{2}$.

We add the shell correction $\delta E(\alpha)$ to the LDM energy. The phenomenological model ${ }^{26}$ is adapted after Reference 24 . At a given deformation we calculate the volumes of fragments and the corresponding numbers of nucleons $Z_{i}(\alpha), N_{i}(\alpha)(i=1,2)$, proportional to the volume of each fragment. Then we add for each fragment the contribution of protons and neutrons

$$
\delta E(\alpha)=\sum_{i} \delta E_{i}(\alpha)=\sum_{i}\left[\delta E_{p i}(\alpha)+\delta E_{n i}(\alpha)\right]
$$

given by

$$
\delta E_{p i}=C s\left(Z_{i}\right) ; \quad \delta E_{n i}=C s\left(N_{i}\right)
$$

where

$$
s(Z)=F(Z) / Z^{-2 / 3}-c Z^{1 / 3}
$$

and similar equation for $s(N)$.

$$
F(n)=\frac{3}{5}\left[\frac{N_{i}^{5 / 3}-N_{i-1}^{5 / 3}}{N_{i}-N_{i-1}}\left(n-N_{i-1}\right)-n^{5 / 3}+N_{i-1}^{5 / 3}\right]
$$

where $n \in\left(N_{i-1}, N_{i}\right)$ is the current number of protons $(Z)$ or neutrons $(N)$ and $N_{i-1}, N_{i}$ are the nearest magic numbers. The parameters $c=0.2, C=6.2 \mathrm{MeV}$ were determined by fit to experimental masses and deformations. The dependence on deformation $\alpha$ is given by Reference 27

$$
\delta E(\alpha)=\frac{C}{2}\left\{\sum_{i}\left[s\left(N_{i}\right)+s\left(Z_{i}\right)\right] \frac{L_{i}(\alpha)}{R_{i}}\right\}
$$

where $L_{i}(\alpha)$ are the lengths of fragments along the symmetry axis. During the deformation process, the variation of separation distance between centers, $\alpha$, induces the variation of the geometrical quantities and of the corresponding nucleon numbers. Each time a proton or neutron number reaches a magic value, the correction energy passes through a minimum, and it has a maximum at midshell.

The integration method used to solve eq 4 is based on the weak dependence of Coulomb energy on the nuclear shape. It is invariant under subtraction from $V_{s}$ of a linear function because $\lambda_{1}$ and $\lambda_{2}$ are arbitrary constants. The extremal surface depends on the quantity with which the Coulomb potential on the nuclear surface differs from the function $\lambda_{1}+\lambda_{2}|z|$, where the constants $\lambda_{1}, \lambda_{2}$ could be chosen in a way to minimize this difference. In the next iteration one uses the solution $\rho(z)$ previously determined.

The following boundary conditions have to be fulfilled

$$
\rho\left(z_{1}\right)=\rho\left(z_{2}\right)=0
$$

$$
\lim _{z \rightarrow z_{1}} d \rho(z) / d z=\infty ; \quad \lim _{z \rightarrow z_{2}} d \rho(z) / d z=-\infty
$$

where $z_{1}$ and $z_{2}$ are the intercepts with $z$ axis at the two tips. Equation 16 are called transversality conditions. For reflection symmetric shapes $z_{1}=-z_{2}=-z_{p}$, hence one can consider only positive values of $z$ in the range $\left(0, z_{p}\right)$. In order to get rid of singularities in eq 16 it is convenient to introduce a new function $u(v)$ substituting $\rho(z)$

$$
u(v)=\mathcal{A}^{2} \rho^{2}(z(v))
$$

where

$$
z(v)=z_{p}-v / \mathcal{A}
$$

$d z / d v=-1 / \mathcal{A}, u^{\prime}=d u / d v=2 \mathcal{A}^{2} \rho(d \rho / d z) d z / d v=-2 \mathcal{A} \rho \rho^{\prime}$, $\rho=\sqrt{u} / \mathcal{A}, u^{\prime 2}=4 u \rho^{\prime 2}, 1+\rho^{\prime 2}=u^{\prime 2} /(4 u)+1, u^{\prime \prime}=d^{2} u / d v^{2}=$ $d\left(u^{\prime}\right) / d v=-2 \mathcal{A}[d \rho(z(v)) / d v] d \rho / d z-2 \mathcal{A} \rho d(\rho(z(v)) / d z=$ $-2 \mathcal{A}(d \rho / d z)(d z / d v)(d \rho / d z)-2 \mathcal{A} \rho\left(d^{2} \rho / d z^{2}\right) d z / d v=2 \rho^{\prime 2}+$ $2 \rho \rho^{\prime \prime}$. By substituting into eq 4 one has

$$
\begin{aligned}
u^{\prime \prime}-2-\frac{1}{u}\left\{u^{\prime 2}+\right. & \left(\frac{5 X V_{s}}{2 \mathcal{A}}+\frac{\lambda_{1}+\lambda_{2} z_{p}}{4 \mathcal{A}}\right. \\
& \left.\left.-\frac{\lambda_{2} v}{4 \mathcal{A}^{2}}\right)\left(4 u+u^{\prime 2}\right)^{3 / 2}\right\}=0
\end{aligned}
$$

Now we can introduce a linear function of $v$ by adding and subtracting $a+b v$ to $5 X V_{s} / 2 \mathcal{A}$ and define $V_{s d}$ as deviation of Coulomb potential at the nuclear surface from a linear function of $v$

$$
V_{s d}=\frac{5 X}{2 \mathcal{A}} V_{s}-a-v b
$$

in which the linear term may be considered an external potential of deformation

$$
\begin{gathered}
a=\frac{5 X}{2 \mathcal{A}} V_{s}(v=0), \\
b=\left[\frac{5 X}{2 \mathcal{A}} V_{s}\left(v=v_{p}\right)-a\right] / v_{p} .
\end{gathered}
$$

Consequently one has

$$
\begin{aligned}
u^{\prime \prime}-2- & \frac{1}{u}\left\{u^{\prime 2}+\left[\left(\frac{\lambda_{1}+\lambda_{2} z_{p}}{4 \mathcal{A}}+a\right)\right.\right. \\
+ & \left.\left(b\left(b-\frac{\lambda_{2}}{4 \mathcal{A}^{2}}\right)+V_{s d}\right]\left(4 u+u^{\prime 2}\right)^{3 / 2}\right\}=0 .
\end{aligned}
$$

As one can see from eq 23 , there are new constants $\mathcal{A}, z_{p}$ related to eq 17 , besides the previous ones $\lambda_{1}$ and $\lambda_{2}$. Nevertheless the solution of eq 23 is not dependent on each parameter; important are the linear coefficients in $v$ of the binomial term within parentheses. By equating with 1 the coefficient of $v$, one can establish the following link between parameter $\mathcal{A}$ and the Lagrange multiplier $\lambda_{2}$

$$
\mathcal{A}^{2}=\lambda_{2} / 4(b-1) \text {. }
$$

In this way $u(v)$ is to be determined by equation

$$
u^{\prime \prime}=2+\frac{1}{u}\left[u^{\prime 2}+\left(v-d+V_{s d}\right)\left(4 u+u^{\prime 2}\right)^{3 / 2}\right]
$$

containing a single parameter $d$. From eq 15,17 one can deduce at the limit

$$
u(0)=0, \quad u^{\prime}(0)=1 / d
$$

and eq 16 is satisfied if $z_{p}=v_{p} / \mathcal{A}$ is obtained from

$$
u^{\prime}\left(v_{p n}\right)=0 \text {. }
$$

The subscript $n$ was introduced as a consequence of the fact that the number of points $v_{p n}$ (depending on $d$ and other parameters), satisfying eq 27 is larger than unity. In other words the function $u(v)$ has the multiplicity of extremes and the subscript $n$ 
counts the number of extremes. To various values of $n$ correspond branches of solution of eq 25 , describing different classes of shapes. Thus for $n=1$ there is no neck, for $n=2$ there is one neck, $n=3$ gives two necks, etc. In order to solve eq 25 one starts with given values of parameters $d$ and $n$. For reflection symmetric shapes $d_{L}=d_{R}$ and $n_{L}=n_{R}$. Although parameter $\mathcal{A}$ is not present in eq 25 we have to know it in order to obtain the shape function 17 . From the volume conservation one has

$$
\mathcal{A}=\left\{\frac{3}{2} \int_{0}^{v_{p n}} u(v) d v\right\}^{1 / 3}
$$

After solving the integro-differential equation one can find the deformation parameter $\alpha=z_{L}^{c}+z_{R}^{c}$, where

$$
z_{L}^{c}=\int_{z_{1}}^{0}|z| \rho^{2}(z) d z / \int_{z_{1}}^{z_{2}} \rho^{2}(z) d z=\frac{3}{2} \mathcal{A}^{-4} \int_{0}^{v_{p}}\left(v_{p}-v\right) u(v) d v
$$

depends on $d$. From $\alpha(d)$, one can obtain the inverse function $d=d(\alpha)$.

For reflection asymmetrical shapes we need to introduce another constraint: the asymmetry parameter, $\eta$, defined by

$$
\eta=\frac{M_{L}-M_{R}}{M_{L}+M_{R}}=\frac{A_{1}-A_{2}}{A_{1}+A_{2}}
$$

should remain constant during variation of the shape function $\rho(z)$. Consequently eq 25 should be written differently for left hand side and right hand side. Now $d_{L}$ is different from $d_{R}$, and so are the parameters $\mathcal{A}_{L}$ and $\mathcal{A}_{R}$. They have to fulfil matching conditions $\rho_{L}(z=0)=\rho_{R}(z=0)$ hence

$$
u_{L}^{1 / 2}\left(v_{p}\right) / \mathcal{A}_{L}=u_{R}^{1 / 2}\left(v_{p}\right) / \mathcal{A}_{R}
$$

The similar condition for derivatives $\rho^{\prime}(z)$ in $z=0, \rho_{L}^{\prime}(z=0)=$ $\rho_{R}^{\prime}(z=0)=0$, is automatically satisfied due to eq 27 . The second derivative $\rho^{\prime \prime}(z)$ can have a discontinuity in $z=0$ if $d_{L} \neq d_{R}$. The parameters $\mathcal{A}_{L}$ and $\mathcal{A}_{R}$ are easily expressed in terms of $\eta$, if we write eq 30 as

$$
\begin{aligned}
& M_{L}=\frac{2 \pi}{3}(1+\eta)=\pi \mathcal{A}_{L}^{-3} \int_{0}^{v_{p}} u_{L}(v) d v, \\
& M_{R}=\frac{2 \pi}{3}(1-\eta)=\pi \mathcal{A}_{R}^{-3} \int_{0}^{v_{p}} u_{R}(v) d v .
\end{aligned}
$$

We assume that $M_{L}+M_{R}$ is equal to the mass of a sphere with $R=1$. From eq 32,33 we obtain

$$
\begin{aligned}
& \mathcal{A}_{L}=(1+\eta)^{-1 / 3} \mathcal{A}_{L 0}, \\
& \mathcal{A}_{R}=(1-\eta)^{-1 / 3} \mathcal{A}_{R 0}
\end{aligned}
$$

where we introduced notations similar to eq 28

$$
\mathcal{A}_{L(R 0)}=\left\{\frac{3}{2} \int_{0}^{v_{p}} u_{L(R)}(v) d v\right\}^{1 / 3}
$$

The shape of a nucleus with given mass asymmetry, $\eta$, is completely determined by eq 34-36; the quantities $u_{L}\left(v_{p}\right)$ and $u_{R}\left(v_{p}\right)$ are obtained as solutions of the differential eq 25 . Again, the deformation $\alpha$ is the distance between centers of mass of the left hand side and right hand side.

There is an alternative way to obtain asymmetric shapes. When we previously discussed the energy minimization with the constraint of deformation conservation, we observed that besides symmetrical solutions for which $d_{L}=d_{R}$ and $n_{L}=n_{R}\left(n_{L(R)}\right.$ is the number of solutions of eq 27 for the left hand side and right hand side), there are also solutions with $n_{L} \neq n_{R}$, meaning shapes with different number of necks on the two sides separated by the plane $z=0$. The parameters $d_{L}$ and $d_{R}$ are linked through the
TABLE 1: Mass number of the heavy fragment corresponding to the minimum of the saddle-point energies in Figure 4.

\begin{tabular}{cccc}
\hline Nuclei & $d_{L}-d_{R}$ & $\eta$ & $A_{1}$ \\
\hline${ }^{238} \mathrm{U}$ & 0.04 & 0.04988 & 124.93 \\
${ }^{232} \mathrm{Th}$ & 0.06 & 0.07517 & 124.72 \\
${ }^{228} \mathrm{Th}$ & 0.08 & 0.09512 & 124.84 \\
\hline
\end{tabular}

condition of continuity of the second derivative $\rho^{\prime \prime}(z)$ in $z=0$, which (using eq 25) may be written as

$$
\left(d_{L}-v_{p L}\right) u_{L}^{1 / 2}\left(v_{p L}\right)=\left(d_{R}-v_{p R}\right) u_{R}^{1 / 2}\left(v_{p R}\right) .
$$

This equation establishes the relationship between $d_{L}$ and $d_{R}$. Unlike the first approach in which $d_{L}$ and $d_{R}$ are free parameters determining $\alpha$ and $\eta$, this time we have a class of shapes with only one parameter; at a given deformation, the mass asymmetry is perfectly determined. This is a drawback when we intend to study the variation of deformation energy with $\alpha$ and $\eta$. Moreover, these kind of shapes have a low chance to be met in practice because of higher value of the energy compared to symmetrical shapes with the same parameter $d$. Generally speaking the solutions of the variational problem are obtained for independent values (not related by eq 37) of parameters $n_{L} \neq n_{R}, d_{L}$ and $d_{R}$. It should be noted that the energies of the corresponding shapes will be higher than those of shapes with the same values of $d_{L}$ and $d_{R}$ but with $n_{L}=n_{R}$.

In order to solve the eq 25 we employ the method of successive approximations. In the first iteration one obtains the solution of differential equation in which Coulomb potential at the nuclear surface is assumed to be a linear function of $z$ (or $v$ ), i.e. if one assumes initially $V_{s}=0$. After solving the equation in such a manner, one calculates the parameters $\mathcal{A}$, $a, b$, which depend on the Coulomb potential and its deviation $V_{s d}$ from a linear function. The quantity $V_{s d}$ obtained in such a way is introduced in eq 25 and the whole procedure is repeated until the desired accuracy is reached. In every iteration the equation is solved with the 2 nd order Runge-Kutta method with constant integration step. The initial value $u^{\prime \prime}(v=0)$ can be found straightforwardly from eq 25 by removing the indetermination in the point $v=0, u^{\prime \prime}(0)=-2+(1-b+g) / 2 d^{2}$, where $g=[5 X /(2 \mathcal{A})]\left[d V_{s}(v) / d v\right]_{v=0}$. The differential equation is integrated up to the point $v=v_{p n}$, in which the first derivative $u^{\prime}\left(v_{p n}\right)$ vanishes. The number $n$ of extremal values of $u(v)$ (equal to the number of necks plus one unit) is an external parameter. The value $v_{p n}$ was determined by linear interpolation between two neighbouring points in which $u^{\prime}$ has opposite signs.

\section{Results}

In Figure 1 we present reflection symmetric nuclear shapes for binary fission of a nucleus with the fissility parameter $X=$ 0.6 (e.g. ${ }^{170} \mathrm{Yb}$ ), obtained for $n_{L}=n_{R}=2$ (one neck), $d_{L}=d_{R}=$ $1.4,1.5,1.7$, and 1.91 (for which $\alpha=1.314,1.644,2.100$, and 2.304 ) and a vanishing mass asymmetry $\eta=0$. The saddle point (maximum value of the conditioned deformation energy minimum) is obtained for $d_{L}=1.91$, at which the shape is deformed and necked-in. The deformation, $\alpha$ (distance between the mass centers of fragments in units of radius of the spherical parent nucleus, $R_{0}$ ), was given above.

A comparison between three nuclear shapes at the saddle point for nuclei with fissilities $X=0.60,0.70$, and 0.82 (corresponding to ${ }^{170} \mathrm{Yb},{ }^{204} \mathrm{~Pb}$, and ${ }^{252} \mathrm{Cf}$ nuclei lying on the line of $\beta$ stability) is presented in Figure 2 . One can see how the necking-in and the elongation are decreasing $(\alpha=2.304,1.822$, and 1.165) when fissility increases from $X=0.60$ to $X=0.82$, in agreement with Reference 4 . In the limit $X=1$ the saddle point shape is spherical.

Within LDM a nonzero mass asymmetry parameter (see the shapes from Figure 3) leads to a deformation energy which increases with $\eta$ as is illustrated in Figure 4, where $\eta$ is replaced 


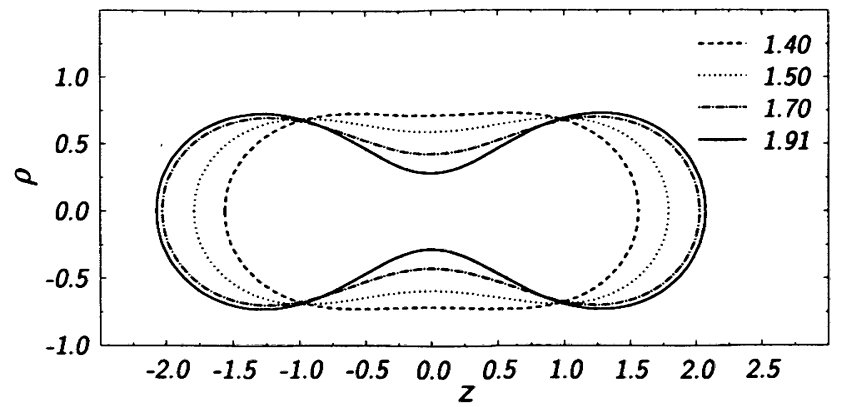

Figure 1. Reflection symmetric nuclear shapes obtained by solving the integro-differential equation for the following values of parameters: $n_{L}=n_{R}=2 ; d_{L}=d_{R}=1.4,1.5,1.7$, and 1.91 (for which the deformations in units of $R_{0}$ are $\alpha / R_{0}=1.314,1.644,2.100$, and 2.304) at $\eta=0$. Binary fission of a nucleus with the fissility $X=0.60$ (e.g. ${ }^{170} \mathrm{Yb}$ ).

by an almost linear dependent quantity $\left(d_{L}-d_{R}\right)$. The reflection asymmetric shapes plotted in Figure 3 , resulted by choosing the input parameters as follows: $n_{L}=n_{R}=2 ; d_{L}=1.40,1.45$, 1.50 , and 1.60 while $d_{R}=1.40$ was kept constant, and so was $X=0.60$. The increasing deformation energy with mass asymmetry in Figure 4, refers to different values of fissilities, namely $X=0.769, X=0.754$, and $X=0.758$ for ${ }^{238} \mathrm{U},{ }^{232} \mathrm{Th}$, and ${ }^{228} \mathrm{Th}$, respectively. By adding the shell corrections $\delta E$ to the LDM deformation energy, $E_{\mathrm{def}}=E_{\mathrm{LDM}}+\delta E$, we succeeded to obtain the minima shown in Figure 4 at a $d_{L}-d_{R}$ of about 0.04,0.06, and 0.08 for ${ }^{238} \mathrm{U},{ }^{232} \mathrm{Th}$, and ${ }^{228} \mathrm{Th}$ nuclei. Interestingly enough, the mass number of the heavy fragment which corresponds to these minima is the same, namely almost $A_{1}=125$ (more exactly $124.93,124.72$, and 124.84 ). One has indeed the results presented in Table 1.

On the other hand, for experimentally determined mass asymmetry ${ }^{28,29}$ the maximum of the fission fragment mass distributions is centered on $A_{1}=140$ in a broad range of mass numbers of parent nuclei. Qualitatively we obtain for the binary cold fission a similar behaviour. Nevertheless, the numerical discrepancy (125 instead of 140) remains to be explained.

The next result presented in Figure 5 refers to asymmetrical shapes with multiple necks which are obtained for $n_{L}=4$ and $n_{R}=2$. At a given value of $d_{L}=2.39,2.44$, and 2.47 one has

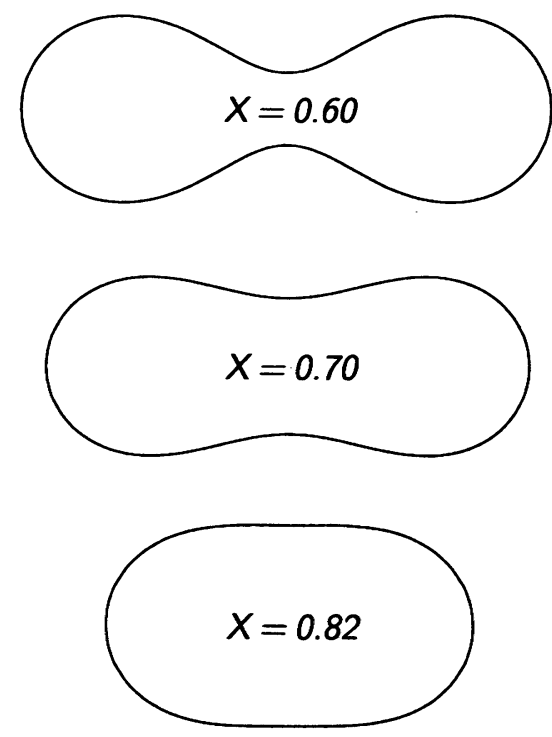

Figure 2. Saddle-point nuclear shapes obtained by solving the integrodifferential equation for the following values of parameters: $n_{L}=n_{R}=$ $2 ; d_{L}=d_{R}=1.91,1.55$, and 1.38 and the binary fission fissilities $X=$ $0.60,0.70$, and 0.82 (for which the deformation $\alpha / R_{0}=2.304,1.822$, and 1.165 ) at $\eta=0$.

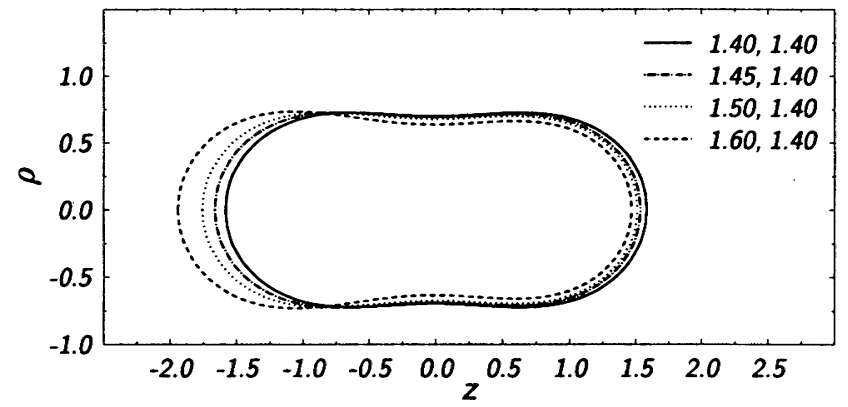

Figure 3. Nuclear shapes (one reflection symmetric and three reflection asymmetric) obtained by solving the integro-differential equation for the following values of parameters: $n_{L}=n_{R}=2 ; d_{L}=1.40,1.45$, 1.50 , and 1.60 keeping $d_{R}=1.40$ and binary fissility $X=0.60$. The mass asymmetry $\eta$ was automatically changed in such a way.

$d_{R}=1.40,1.43$, and 1.46 . The corresponding deformation parameters in units of $R_{0}$ are $1.840,1.977$, and 2.060, respectively. The total deformation energy in units of $E_{s}^{0}$ increases from 0.135 to 0.148 and 0.152 .

The elongated shapes for ternary and quaternary fissions are shown in Figures 6 and 7. For shapes with three fragments and two necks $\left(n_{L}=n_{R}=3\right.$, see Figure 6), by increasing $d_{L}=d_{R}$, from 2.25 to 2.80 and 7.00 the deformation increases from 1.650 to 2.306 and 2.730 . In the same time the elongation is initially increased from 5.234 to 5.392 and then decreased to 5.24; the fragment radii are $0.461 / 0.814 / 0.461$, $0.592 / 0.753 / 0.592$, and $0.673 / 0.659 / 0.673$, leading to decreasing energies in units of $E_{s}^{0}$ from 0.165 to 0.150 and 0.134 . The last configuration with $E / E_{s}^{0}=0.134$ is not far from a "true ternary-fission" in which the three fragments are almost identical: ${ }_{70}^{170} \mathrm{Yb} \rightarrow{ }_{23}^{56} \mathrm{~V}+{ }_{23}^{56} \mathrm{~V}+{ }_{24}^{58} \mathrm{Cr}$ and the $Q$ value is $83.639 \mathrm{MeV}$. One may compare the above $E / E_{s}^{0}$ value with the touching-point energy of these spherical fragments $\left(E_{t}-Q\right) / E_{s}^{0}=0.239$. It is larger, as expected, because of the finite neck of the shapes in Figure 6. For $\alpha$-accompanied fission of ${ }^{170} \mathrm{Yb}$ with two ${ }_{34}^{83} \mathrm{Se}$ fragments $Q=87.484 \mathrm{MeV}$ is larger and the touching point energy $\left(E_{t}-Q\right) / E_{s}^{0}=0.103$ is lower. A lower $Q=70.859 \mathrm{MeV}$ and higher energy barrier $\left(E_{t}-Q\right) / E_{s}^{0}=0.147$ is obtained for ${ }^{10} \mathrm{Be}$ accompanied fission of ${ }^{170} \mathrm{Yb}$ with ${ }_{33}^{80} \mathrm{As}$ fission fragments. One should stress that we present shapes in Figures 1, 3, 5-7 which are produced for various values of input parameters; only one of these shapes in every figure corresponds to the saddlepoint. One should not be confused about the unexpected shape with $d_{L}=d_{R}=2.25$ in Figure 6 having a large fragment between two smaller ones; it was produced due to the low value of $d_{L}=d_{R}$.

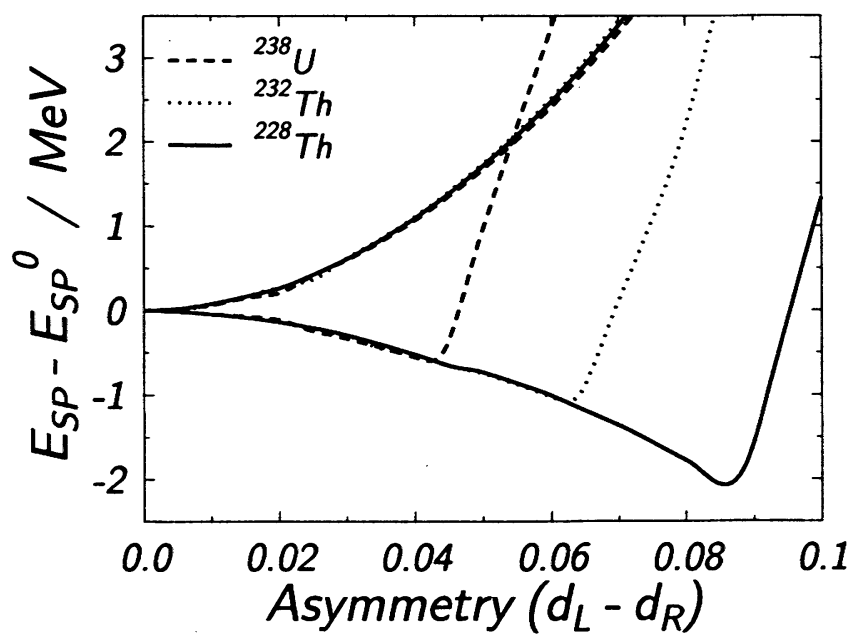

Figure 4. Saddle-point deformation energy versus mass asymmetry parameter for binary fission of ${ }^{238} \mathrm{U}$ and ${ }^{232,228} \mathrm{Th}$ nuclei. One can see a monotonous increase within a pure liquid drop model and minima when the shell corrections are included. 


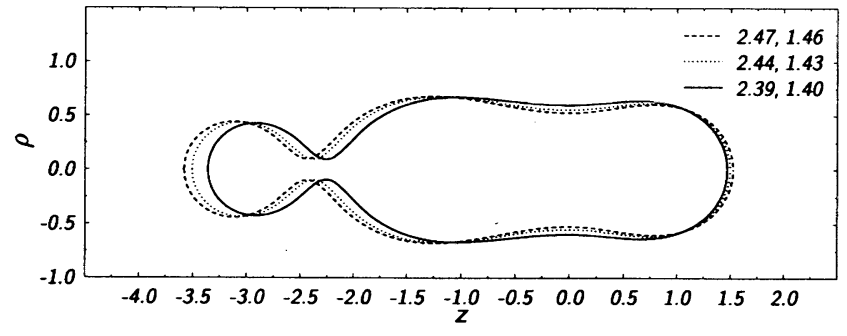

Figure 5. An alternative way to generate asymmetrical shapes in which $n_{L} \neq n_{R}$ illustrated for $n_{L}=4$ and $n_{R}=2$. At a given value of $d_{L}=2.39$, 2.44 , and 2.47 one obtains $d_{R}=1.40,1.43$, and 1.46 . The binary fissility $X=0.60$.

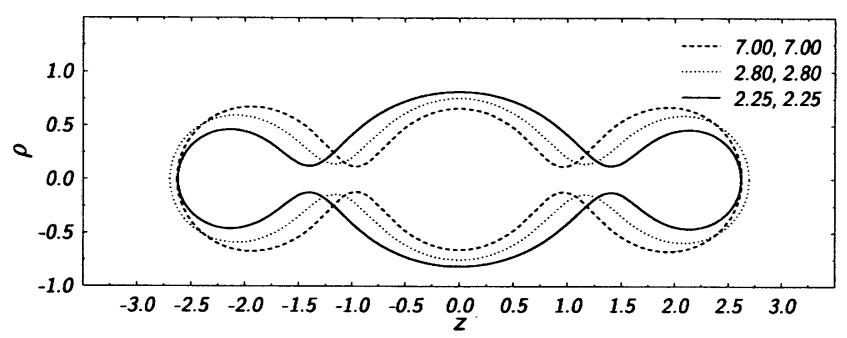

Figure 6. Nuclear shapes obtained by solving the integro-differential equation for $n_{L}=n_{R}=3$ and $d_{L}=d_{R}=2.25,2.80$, and 7.00. The binary fissility $X=0.60$.

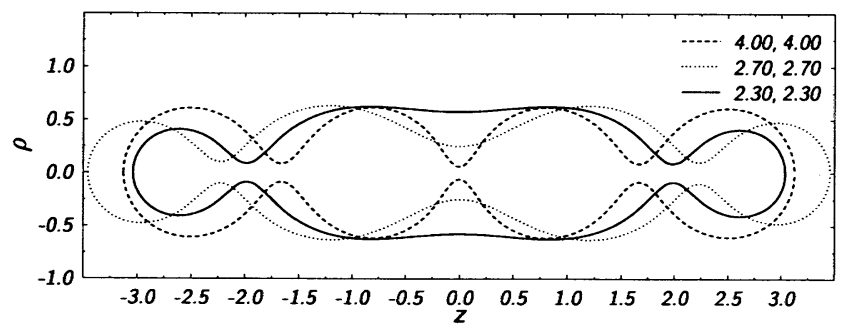

Figure 7. Nuclear shapes obtained by solving the integro-differential equation for $n_{L}=n_{R}=4$ and $d_{L}=d_{R}=2.30,2.70$, and 4.00. The binary fissility $X=0.60$.

The shapes with four fragments and three necks $\left(n_{L}=\right.$ $\left.n_{R}=4\right)$ can be seen in Figure 7. When $d_{L}=d_{R}$ increases from 2.30 to 2.70 and 4.00 the deformation takes the values $2.144,3.136$, and 3.233 and the elongations are $6.077,6.916$, and 6.252. The fragment radii are $0.408 / 0.625 / 0.625 / 0.408$, $0.479 / 0.632 / 0.632 / 0.479$, and $0.608 / 0.616 / 0.616 / 0.608$, for which the energies in units of $E_{s}^{0}$ are $0.188,0.216$, and 0.214 , respectively. The last shape, with $E / E_{s}^{0}=0.214$ approaches a fission into almost identical four fragments ${ }_{70}^{170} \mathrm{Yb} \rightarrow{ }_{17}^{42} \mathrm{Cl}+{ }_{17}^{42} \mathrm{Cl}+{ }_{18}^{43} \mathrm{Ar}+{ }_{18}^{43} \mathrm{Ar}$. Again the configuration with aligned spherical fragments in touch is higher in energy: $\left(E_{t}-Q\right) / E_{s}^{0}=0.324$. Even more complex shapes can be obtained by further increasing the values of $n_{L}=n_{R}$.

\section{Conclusions}

The method of finding the most general axially-symmetric shape at the saddle point without introducing apriori a parametrization (inherently limited due to the finite number of deformation coordinates), by solving an integro-differential equation was tested for binary, ternary, and quaternary fission processes within a pure liquid drop model.

It proved its practical capability in what concerns fission into two, three, or four identical fragments, for which fission barriers given by shapes with rounded necks are, as expected, lower than those of aligned spherical fragments in touch.

Nevertheless, in the absence of any shell correction it is not possible to reproduce the experimental data, or to give results for particle-accompanied fission.

By adding (phenomenological) shell corrections we suc- ceeded to obtain the minima shown in Figure 4 at a finite value of mass asymmetry for the binary fission of ${ }^{238} \mathrm{U},{ }^{232} \mathrm{Th}$, and ${ }^{228} \mathrm{Th}$ nuclei. Moreover, the mass number of the heavy fragment which corresponds to these minima is the same $A_{1} \simeq 125$. A discrepancy (if any because our result was obtained in the absence of dissipation, hence it refers to cold fission) remains to be explained in the future.

Acknowledgement. This work was partly supported by the Center of Excellence IDRANAP under contract ICA1-CT-200070023 with European Commission, Brussels, by Bundesministerium für Bildung und Forschung (BMBF), Bonn, Gesellschaft für Schwerionenforschung (GSI), Darmstadt, by Japanese Society for the Promotion of Science (JSPS), Tokyo, by the Japan Atomic Energy Research Institute (JAERI), Tokai, and by the Ministry of Education and Research, Bucharest. One of us (DNP) would like to acknowledge the hospitality and financial support received during his research stage in Frankfurt am Main and Tokai, when the present work was completed.

\section{References}

(1) J. M. Eisenberg and W. Greiner, Nuclear Theory. Vol. I Nuclear Models. Vol. II Nuclear Excitations. Vol. III Microscopic Theory (North-Holland, Amsterdam, 1987).

(2) Nuclear Decay Modes, edited by D. N. Poenaru, (IOP Publishing, Bristol, 1996); Handbook of Nuclear Properties, edited by D. N. Poenaru and W. Greiner (Clarendon Press, Oxford, 1996).

(3) A. Iwamoto, Z. Phys. A 349, 265 (1994).

(4) S. Cohen and W. J. Swiatecki, Ann. Phys. (N.Y.) 22, 406 (1963).

(5) V. M. Strutinsky, JETF 42, 1571 (1962).

(6) D. L. Hill, Proceedings of the 2nd U.N. International Conference on the Peaceful Uses of Atomic Energy (United Nations, Geneva, 1958), p. 244.

(7) M. Brack, J. Damgaard, A. Jensen, H. C. Pauli, V. M. Strutinsky, and G. Y. Wong, Rev. Mod. Phys. 44, 320 (1972).

(8) W. Greiner and J. A. Maruhn, Nuclear Models (Springer, Berlin, 1996); J. A. Maruhn and W. Greiner, Phys. Rev. Lett. 32, 548 (1974).

(9) I. Muntian, Z. Patyk, and A. Sobiczewski, J. Nucl. Radiochem. Sci. 3, 169 (2002).

(10) W. D. Myers and W. J. Swiatecki, Nucl. Phys. A 601, 141 (1996).

(11) P. Möller and A. Iwamoto, Phys. Rev. C 61, 047602 (2000); P. Möller, D. G. Madland, A. J. Sierk, and A. Iwamoto, Nature 409, 785 (2001).

(12) U. Brosa, S. Grossmann, and A. Müller, Phys. Rep. 197, 167 (1990).

(13) A. Gavron, Phys. Rev. C 11, 580 (1975).

(14) T. Ohtsuki, Y. Nagame, and H. Nakahara, Heavy Elements and Related New Phenomena, edited by W. Greiner and R. K. Gupta (World Scientific, Singapore, 1999), p. 507.

(15) Y. Nagame, I. Nishinaka, K. Tsukada, Y. Oura, S. Ichikawa, H. Ikezoe, Y. L. Zhao, K. Sueki, H. Nakahara, M. Tanikawa, T. Ohtsuki, H. Kudo, Y. Hamajima, K. Takamiya, and Y. H. Chung, Phys. Lett. B 387, 26 (1996); I. Nishinaka, Y. Nagame, K. Tsukada, H. Ikezoe, K. Sueki, H. Nakahara, M. Tanikawa, and T. Ohtsuki, Phys. Rev. C 56, 891 (1997).

(16) Y. L. Zhao, Y. Nagame, I. Nishinaka, K. Sueki, and H. Nakahara, Phys. Rev. C 62, 014612 (2000).

(17) Y. L. Zhao, I. Nishinaka, Y. Nagame, M. Tanikawa, K. Tsukada, S. Ichikawa, K. Sueki, Y. Oura, H. Ikezoe, S. Mitsuoka, H. Kudo, T. Ohtsuki, and H. Nakahara, Phys. Rev. Lett. 82, 3408 (1999).

(18) J. H. Hamilton, A. V. Ramayya, J. Kormicki, W. C. Ma, Q. Lu, D. Shi, J. K. Deng, S. J. Zhu, A. Sandulescu, W. 
Greiner, G. M. Ter-Akopian, Yu. Ts. Oganessian, G. S. Popeko, A. V. Daniel, J. Kliman, V. Polhorsky, M. Morhac, J. D. Cole, R. Aryaeinejad, I. Y. Lee, N. R. Johnson, and F. K. McGowan, J. Phys. G 20, L85 (1994); J. H. Hamilton, A. V. Rammaya, S. J. Zhu, G. M. Ter-Akopian, Yu. Ts. Oganessian, J. D. Cole, J. O. Rasmussen, and M. A. Stoyer, Prog. Part. Nucl. Phys. 35, 635 (1995).

(19) A. V. Ramayya, J. H. Hamilton, J. K. Hwang, L. K. Peker, J. Kormicki, B. R. S. Babu, T. N. Ginter, A. Sandulescu, A. Florescu, F. Carstoiu, W. Greiner, G. M. Ter-Akopian, Yu. Ts. Oganessian, A. V. Daniel, W. C. Ma, P. G. Varmette, J. O. Rasmussen, S. J. Asztalos, S. Y. Chu, K. E. Gregorich, A. O. Macchiavelli, R. W. Macleod, J. D. Cole, R. Aryaeinejad, K. Butler-Moore, M. W. Drigert, M. A. Stoyer, L. A. Bernstein, R. W. Lougheed, K. J. Moody, S. G. Prussin, S. J. Zhu, H. C. Griffin, and R. Donangelo, Phys. Rev. C 57, 2370 (1998); A. V. Ramayya, J. K. Hwang, J. H. Hamilton, A. Sandulescu, A. Florescu, G. M. Ter-Akopian, A. V. Daniel, Yu. Ts. Oganessian, G. S. Popeko, W. Greiner, J. D. Cole, and GANDS95 Collaboration, Phys. Rev. Lett. 81, 947 (1998).

(20) D. N. Poenaru and W. Greiner, J. Phys. G 25, L7 (1999); D. N. Poenaru, W. Greiner, J. H. Hamilton, A. V. Ramayya,
E. Hourany, and R. A. Gherghescu, Phys. Rev. C 59, 3457 (1999); D. N. Poenaru, W. Greiner, J. H. Hamilton, and A. V. Ramayya, J. Phys. G 27, L19 (2001).

(21) D. N. Poenaru, B. Dobrescu, W. Greiner, J. H. Hamilton, and A. V. Ramayya, J. Phys. G 26, L97 (2000); Advances in Nuclear Physics, edited by D. N. Poenaru and S. Stoica (World Scientific, Singapore, 2000), p. 91.

(22) K. T. R. Davies and A. J. Sierk, Phys. Rev. C 31, 915 (1985).

(23) R. A. Gherghescu, D. N. Poenaru, and W. Greiner, Z. Phys. A 354, 367 (1996).

(24) W. D. Myers and W. J. Swiatecki, Nucl. Phys. A 81, 1 (1966).

(25) D. N. Poenaru and M. Ivaşcu, Comput. Phys. Commun. 16, 85 (1978).

(26) D. N. Poenaru, W. Greiner, and R. A. Gherghescu, J. Phys. G 24, L23 (1998).

(27) H. Schultheis and R. Schultheis, Phys. Lett. B 37, 467 (1971).

(28) H. R. von Gunten, Actinides Rev. 1, 275 (1969).

(29) E. A. C. Croach, At. Data Nucl. Data Tables 19, 417 (1977).

(30) D. L. Hill, Phys. Rev. 78, 197 (1950). 